\title{
ON SIMILARITY SOLUTIONS AND BLOW-UP SPECTRA FOR A SEMILINEAR WAVE EQUATION
}

\author{
BY \\ V. A. GALAKTIONOV (Department of Mathematical Sciences, University of Bath, Bath BA2 \\ $7 A Y$, UK and Keldysh Institute of Applied Mathematics, Miusskaya Sq. 4, 125047 Moscow, \\ Russia) \\ AND
}

S. I. POHOZAEV (Steklov Mathematical Institute, Gubkina St. 8, 117966 Moscow, Russia)

Abstract. We construct countable spectra of different asymptotic patterns of selfsimilar and approximate self-similar types for global and blow-up solutions for the semilinear wave equation

$$
u_{t t}=\Delta u+|u|^{p-1} u, \quad x \in \mathbf{R}^{N}, t>0,
$$

in different ranges of exponent $p$ and dimension $N$.

1. Introduction. We consider some aspects of asymptotic behaviour and singularity blow-up formation phenomena for the classical semilinear wave equation (SWE, for short)

$$
u_{t t}=\Delta u+|u|^{p-1} u \quad \text { in } \mathbf{R}^{N} \times \mathbf{R}_{+},
$$

where $p>1$ is a fixed exponent. Due to superlinear growth of the lower-order term $|u|^{p-1} u$ for $u \gg 1$, the solution of the Cauchy problem to (1.1) may blow-up in finite time forming blow-up surfaces with finite propagation. Singularity formation phenomena for the SWE is known from the 1950s, starting with Keller's work [25] and moving to John's [20] and Kato's [23] results. We refer to [7], [8], [26], [41], [44], where regularity properties of blow-up surfaces and other asymptotic properties were studied in detail. Extensive lists of references on blow-up solutions of semilinear hyperbolic equations are available in books [1] and [33].

In this paper we study asymptotic behaviour of blowing up and global solutions of (1.1) by constructing self-similar solutions or approximate similarity solutions. In Sections 2

Received February 26, 2002 and, in revised form, April 16, 2002.

2000 Mathematics Subject Classification. Primary 35K55, 35K65.

Key words and phrases. Semilinear wave equation, blow-up, asymptotic behaviour, similarity solutions, fibering method, quadratic pencil, eigenvalue problem.

E-mail address: vag@maths.bath.ac.uk

E-mail address: pohozaev@class.mi.ras.ru 
and 3 we consider globally decaying very singular self-similar solutions of the semilinear equation (1.1) of the form

$$
u_{*}(x, t)=t^{-2 /(p-1)} \theta(\eta), \quad \eta=x / t,
$$

where function $\theta$ satisfies a semilinear degenerate elliptic equation inside and outside the light cone $S_{1}=\{|x|=t\}$. The study of such similarity structures in the radial setting leading to a nonlinear ODE began by Kavian and Weissler [24]. In recent years evolution (PDE) approaches have been developed; see [35]-[37] and [42].

In Sec. 2, we present the results obtained by the fibering method of calculus of variations [39], [40], establishing existence of a countable spectrum of such self-similar solutions to equation (1.1) in $B_{1}=\{|x|<t\}$ in the parameter range

$$
1<p \leq p_{1}=(N+3) /(N-1) .
$$

In the range

$$
p_{1}<p<p_{S}=(N+2) /(N-2),
$$

where $p_{S}$ is the critical Sobolev exponent for the elliptic operator $\Delta u+|u|^{p-1} u$, by using a shooting argument, we prove existence of continuous weak self-similar solutions defined everywhere in $\mathbf{R}^{N} \times \mathbf{R}_{+}$. These solutions are invertible and $u_{*}(x,-t)$ describe finite time blow-ups as $t \rightarrow-0$.

The similarity analysis is directly related to the question on the stability of the trivial stationary solution $u \equiv 0$ which is unstable in the range

$$
1<p \leq p_{c}=\left[N+1+\left(N^{2}+10 N+7\right)^{1 / 2}\right] / 2(N-1) .
$$

Moreover, solutions with arbitrarily small initial data blow-up in finite time and are stable in the supercritical range $p>p_{c}$. It is known that for equation (1.1) in the stability supercritical range $p>p_{c}$, there exists the critical exponent $p_{1}>p_{c}$, and there hold:

(i) For $p \geq p_{1}$, the asymptotic behaviour of small global solutions as $t \rightarrow \infty$ is described by the linear equation

$$
u_{t t}=\Delta u,
$$

with certain initial data, so that the nonlinear term $|u|^{p-1} u$ is negligible for large times; see [30] where a complete list of related references on the preceding results and a historical survey are presented.

(ii) If $p_{c}<p<p_{1}$, then the global solvability for small initial data takes place in the weighted Strichartz functional classes [15].

In different parameter ranges, self-similar solutions of (1.1) play an important role for classes of both global and blow-up solutions of semilinear hyperbolic equations. Moreover, PDE approaches to the existence, uniqueness, and stability of self-similar solutions turned out to be quite effective; see [35]-[37] and [42]. For semilinear and quasilinear heat (parabolic) equations like

$$
u_{t}=\Delta u-|u|^{p-1} u \quad \text { in } \mathbf{R}^{N} \times \mathbf{R}_{+},
$$

evolution constructions of infinite-dimensional subsets of very singular self-similar solutions were performed earlier in [21], [22]. 
Concerning blowing up solutions and types of pointwise formation of singularities, in some parameter ranges, the exact self-similar solutions either do not exist or are not stable in sufficiently wide classes of initial data. Known stability results generate restrictive conditions on sufficiently small initial data which are supposed to be uniformly close to the similarity one. In particular, the subcritical range (1.2) poses difficult open problems.

In Sec. 4 we present another countable spectrum of blow-up patterns for (1.1). These are approximate self-similar solutions obtained by matching involving separable spacetime structures on the stable subspace of a linearized rescaled self-similar operator in the inner region and self-similar solutions of a nonlinear second-order ordinary differential equation in the intermediate region. In this connection, we refer to the asymptotic analysis by Henry [17] for semilinear parabolic equations as Morse-Smale systems, where a time-dependent Sturm-Liouville theory was created and the local structure of multiple zeros of solutions was shown to be governed by Hermite polynomials (this idea goes back to Sturm [45]). Similar linearized blow-up solutions with inner structure governed by Hermite polynomials as eigenfunctions of the operator

$$
\mathbf{A}=\frac{1}{\rho} \nabla \cdot(\rho \nabla): H_{\rho}^{2}\left(\mathbf{R}^{N}\right) \rightarrow L_{\rho}^{2}\left(\mathbf{R}^{N}\right) \quad \text { with weight } \rho=e^{-|x|^{2} / 4},
$$

were obtained in the study of blow-up for semilinear heat equations like

$$
u_{t}=\Delta u+u^{p}, \quad p>1 .
$$

See, e.g., [2], [5], [10], [18], [32], [46], [47], and references therein.

For the SWE (1.1), such a matching construction leads to more difficult eigenvalue problems for quadratic pencils of non-self-adjoint operators, and the corresponding matching procedure becomes much more delicate. This formal part of our asymptotic analysis can be considered as a refined description of singularity spectra already available for SWEs; cf. [26], p. 1891 and [1], p. 6.

2. Self-similar solutions via fibering method. We begin to study the asymptotic behaviour of global and blow-up solutions of the autonomous semilinear equation

$$
u_{t t}=\Delta u+f(u) \quad \text { in } \mathbf{R}^{N} \times \mathbf{R}_{+},
$$

where $f(u)$ is a homogeneous nonlinearity of order $p>1$ :

$$
\text { either } f(u)=|u|^{p} \text { or } f(u)=|u|^{p-1} u \text {. }
$$

2.1. Subcritical Sobolev range. Let $1<p<p_{S}$. As a first step in the asymptotic analysis, we consider the following global self-similar solutions of equation (2.1):

$$
u_{*}(x, t)=t^{-\alpha} \theta(\eta), \quad \eta=x / t, \quad \alpha=2 /(p-1)>0,
$$

where the function $\theta$ solves the following elliptic equation:

$$
\mathbf{A} \theta \equiv \Delta \theta-\nabla(\nabla \theta \cdot \eta) \cdot \eta-(1+2 \alpha) \nabla \theta \cdot \eta-\alpha(1+\alpha) \theta=-f(\theta), \eta \in \mathbf{R}^{N} .
$$

Using the identity

$$
\nabla(\nabla \theta \cdot \eta) \cdot \eta=\sum_{(i, j)} \theta_{\eta_{i} \eta_{j}} \eta_{i} \eta_{j}+\nabla \theta \cdot \eta
$$


the linear operator is written down in the form

$$
\mathbf{A} \theta=\Delta \theta-\sum_{(i, j)} \theta_{\eta_{i} \eta_{j}} \eta_{i} \eta_{j}-2(1+\alpha) \nabla \theta \cdot \eta-\alpha(1+\alpha) \theta .
$$

We begin with the first properties of $\mathbf{A}$ and $\theta$.

Proposition 2.1. The quadratic form of the linear operator (2.4) satisfies

$$
B_{\mathbf{A}}(z, z) \equiv \sum_{(i, j)}\left(\delta_{i j}-\eta_{i} \eta_{j}\right) z_{i} z_{j} \geq|z|^{2}\left(1-|\eta|^{2}\right) .
$$

so that $\mathbf{A}$ is uniformly elliptic on any compact subset from the unit ball $B_{1}$.

Proof. By the Cauchy-Bunyakovskii-Schwarz inequality, we have $B_{\mathbf{A}}(z, z)=|z|^{2}-(z$. $\eta)^{2} \geq|z|^{2}\left(1-|\eta|^{2}\right)$.

Proposition 2.2. Let there exist a sufficiently regular, compactly supported solution $\theta$ of Eq. (2.7). Then

$$
\operatorname{supp} \theta=\bar{B}_{1} .
$$

Proof. Let $\operatorname{supp} \theta \subseteq\{|\eta| \leq c\}$. Then $\operatorname{supp} u_{*}(x, t) \subseteq\{|x| \leq c t\}$, and passing to the limit $t \rightarrow+0$ in $(2.2)$, we see that supp $u_{*}(x,+0)=\{0\}$; i.e., the initial data for $u_{*}(x, t)$ are concentrated at the origin, whence the result by the characteristic propagation. On the other hand, the equality in (2.6) follows by the strong Maximum Principle applied to Eq. (2.3), which is uniformly elliptic in any domain bounded away from the unit sphere $S_{1}=\{|\eta|=1\}$.

Let us look for a solution $\theta$ of the elliptic equation (2.3) with the radially symmetric support $B_{1}$. This suggests to consider this problem in the radial setting, where $\theta$ depends on the single radial variable $\xi=|\eta|$. Then (2.3) reduces to the ODE

$$
\mathbf{A}_{\mathbf{r}} \theta \equiv\left(1-\xi^{2}\right) \theta^{\prime \prime}+\frac{N-1}{\xi} \theta^{\prime}-2(1+\alpha) \theta^{\prime} \xi-\alpha(1+\alpha) \theta=-f(\theta), \quad \xi \neq 1 .
$$

We impose a standard symmetry condition at the origin $\theta^{\prime}(0)=0$ (a natural functional restriction for the radial setting of the Laplacian). The linear operator $\mathbf{A}$ degenerates on the sphere $\xi=|\eta|=1$ which is the light cone $\{|x|=t\}$ for the linear hyperbolic equation. In the global sense, we are looking for a weak solution $\theta(\xi)$ of $(2.7)$ in $\mathbf{R}_{+}$, which, in particular, is continuous at the degeneracy point $\xi=1$.

It is already known that in the critical $p=p_{S}$ and supercritical $p>p_{S}$ Sobolev ranges, nontrivial self-similar solutions with finite-energy $\left(f(\theta)=|\theta|^{p-1} \theta\right)$

$$
E(\theta)=\frac{1}{2}\left\|\frac{2 \theta}{p-1}+\xi \theta^{\prime}\right\|_{2}^{2}+\frac{1}{2}\left\|\theta^{\prime}\right\|_{2}^{2}-\frac{1}{p+1}\|\theta\|_{p+1}^{p+1}
$$

do not exist; see results in [24], where a detailed asymptotic analysis of the ODE (2.7) at the critical points as well as other local and global properties of the solutions are available. We will use some of the results from [24] in our construction of nontrivial solutions in the subcritical Sobolev range. 
2.2. The first spatially flat similarity pattern. Let $f(\theta)=|\theta|^{p-1} \theta$. One can see that Eq. (2.3) or (2.7) admits a constant solution

$$
\theta_{1}(\eta) \equiv c_{*}=[\alpha(1+\alpha)]^{1 /(p-1)} \equiv\left[2(p+1) /(p-1)^{2}\right]^{1 /(p-1)} .
$$

This profile gives the first self-similar pattern

$$
u_{1}(x, t)=t^{-\alpha} c_{*} \text {. }
$$

Obviously, the piecewise pattern $\tilde{\theta}_{1}(\eta)=\left\{c_{*},|\eta| \leq 1 ; 0,|\eta|>1\right\}$, has a strong discontinuity on $S_{1}$ and $\tilde{u}_{1}(x, t)=t^{-\alpha} \tilde{\theta}_{1}(\eta)$ is not a proper weak solution of the semilinear hyperbolic equation in the sense that it does not satisfy the corresponding integral equation given by the D'Alambert-Poisson-Kirchhoff formulae. In order to choose a proper solution of hyperbolic equations with a priori known singularity propagation, the concept of the wave front set in the microlocal analysis applies; see Chapter 8 in [19]. On the other hand, such a discontinuous solution $\tilde{u}_{1}$ cannot be obtained as a limit of a sequence of global regular solutions $\left\{u_{\varepsilon}\right\}$ obtained by a suitable truncation of the equation, $u=\lim _{\varepsilon \rightarrow 0} u_{\varepsilon}$, with continuous (truncated) initial data $u_{0 \varepsilon}, u_{1 \varepsilon} \rightarrow u_{0}, u_{1}$ as $\varepsilon \rightarrow 0$ in a certain metric (say, in $L^{1}$ ). Such an extended semigroup theory is natural in singular blow-up parabolic problems; see [14], Sec. 3 and references therein.

2.3. Very singular self-similar solution (VSS). Assuming that a compactly supported continuous profile $\theta$ exists and integrating (2.2) over $\mathbf{R}^{N}$ (or $B_{1}$ ), we obtain

$$
E_{*}(t)=\int_{\mathbf{R}^{N}} u_{*}(x, t) d x=t^{\gamma} c_{*}, \quad c_{*}=\int_{\mathbf{R}^{N}} \theta(\eta) d \eta,
$$

where $\gamma=-2 /(p-1)+N$. Therefore, if $\gamma<0$, i.e., $1<p<p_{F}=1+2 / N$ (here subindex $f$ stays for Fujita; see below) and $c_{*} \neq 0$, then $u_{*}$ is a very singular solution in the sense that it has non-integrable initial data:

$$
u_{*}(x, t) \rightarrow u_{* 0}(x) \notin L^{1}\left(\mathbf{R}^{N}\right) \quad \text { as } t \rightarrow+0 .
$$

On the other hand, in the critical case $p=p_{F}$ we have $u_{* 0}=c_{*} \delta(x)$. In a similar way, one can treat the type of singularity of the derivative $u_{* t}(x, t)=-t^{-\alpha-1}(\alpha \theta+\nabla \theta \cdot \eta)$, so that $u_{* t}(x, t) \rightarrow u_{* 1}(x) \in L_{\text {loc }}^{1}\left(\mathbf{R}^{N}\right)$ as $t \rightarrow+0$ provided that $-\alpha-1+N \geq 0$; i.e., $p \geq p_{K}=(N+1) /(N-1)>p_{F}$, where $p_{K}$ is Kato's critical exponent [23]. If $p=p_{K}$, then $u_{* 1}=c_{1} \delta(x), c_{1} \neq 0$. In the second supercritical range, $p>p_{K}$, self-similar solutions $u_{*} \not \equiv 0$ satisfy the trivial initial conditions $u_{* 0}=0=u_{* 1}$ in $\mathcal{D}^{\prime}\left(\mathbf{R}^{N}\right)$ or $L^{1}\left(\mathbf{R}^{N}\right)$. This means nonuniqueness of solution in a natural sense. It is curious that the exponent $p_{F}$ coincides with the critical Fujita exponent [11] for the semilinear parabolic equation (1.4), where $p_{F}$ determines the subcritical range $p<p_{F}$ and the supercritical one $p>p_{F}$ of unstability and stability of the trivial stationary solution $u \equiv 0$; see Chapter 4 in [43] and an extended list of references therein.

2.4. Properties of the linear operator in the radial setting. In order to construct asymptotic patterns for the SWE, we need some analysis of the linear and linearized degenerate elliptic operators. We begin with the properties of the radial linear operator (2.7) in the unit ball $B_{1}=\{|\eta|<1\}$ which is identified with the interval $\xi \in B_{1}=[0,1)$ for sufficiently smooth even functions. Its Sturm-Liouville form is

$$
\mathbf{A}_{\mathbf{r}}=\frac{1}{\rho}[(d / d \xi)(a d / d \xi)-\alpha(1+\alpha) \rho I]
$$


with positive coefficients on $(0,1)$,

$$
\begin{gathered}
\rho(\xi)=\xi^{N-1}\left(1-\xi^{2}\right)^{\nu}>0, \quad a(\xi)=\xi^{N-1}\left(1-\xi^{2}\right)^{1+\nu}>0, \\
\nu=\alpha-(N-1) / 2=2 /(p-1)-(N-1) / 2,
\end{gathered}
$$

where $\nu>0$ for $1<p<p_{1}$ and $\nu=0$ if $p=p_{1}$. Operator $\mathbf{A}_{\mathbf{r}}$ is symmetric in the weighted class $C_{0 \rho}^{\infty}\left(B_{1}\right)$ of symmetric functions with compact support in $B_{1},-\mathbf{A}_{\mathbf{r}}>0$ is semibounded below and admits a unique Friedrichs extension [4], which is a self-adjoint operator in the weighted Hilbert space $L_{\rho}^{2}\left(B_{1}\right)$ of even functions. Then its domain is a weighted Sobolev space $H_{a}^{2}\left(B_{1}\right)$; see below. This follows from the classical Hardy inequality in the form [16].

Let us describe the spectrum of the linear operator. For the radial setting of the Laplacian, the left-hand singular endpoint $\xi=0$ is in the limit-point case for $N \geq 4$. Indeed, since $\rho \sim a \sim \xi^{N-1}$ as $\xi \rightarrow 0$, we have two possible expansions of the solutions near the singularity: $\theta_{1} \sim 1, \theta_{2} \sim \xi^{2-N}$, and obviously $\theta_{1} \in L_{\rho}^{2}$ and $\theta_{2} \notin L_{\rho}^{2}$, provided that $N \geq 4$. For $N=2$ and $N=3$, we need to impose a natural condition of boundedness at the origin or, which is equivalent, we assume that $\xi^{N-2} \theta(\xi) \rightarrow 0$ as $\xi \rightarrow 0$. In the onedimensional case $N=1$, the origin is not a singular point, where we put the symmetry condition $\theta^{\prime}(0)=0$.

We next put $\xi=\sin \zeta: J=[0, \pi / 2) \rightarrow B_{1}$. Then

$$
\begin{gathered}
\mathbf{A}_{\mathbf{r}}=\frac{1}{\bar{\rho}}[(d / d \xi)(\bar{\rho} d / d \xi)-\alpha(1+\alpha) \bar{\rho} I], \\
\bar{\rho}(\zeta)=\sin ^{N-1} \zeta \cos ^{\mu} \zeta, \quad \mu=1+2 \nu=2 \alpha-(N-2)
\end{gathered}
$$

$\left(\mu>0\right.$ if $\left.p<p_{S}\right) . \mathbf{A}_{\mathbf{r}}$ is symmetric and semibounded in $C_{0 \bar{\rho}}^{\infty}(J)$ and its self-adjoint extension in $L_{\bar{\rho}}^{2}(J)$ has the domain $H_{\bar{\rho}}^{2}(J)$.

Consider the right-hand singular endpoint $\xi=1$, i.e., $\zeta=\pi / 2$. We use representation (2.13). Similar to the analysis at $\xi=0$, using a standard procedure from the theory of ordinary differential operators (see books [3] and [29]) we have to study the asymptotics of two linearly independent solutions near this singular point. Setting for convenience $s=\pi / 2-\zeta$ and using the expansion of the coefficients $\bar{\rho} \sim s^{\mu}$, we have that in the first approximation $\mathbf{A}_{\mathbf{r}} Y \sim s^{-\mu}\left[\left(s^{\mu} Y^{\prime}\right)^{\prime}+\cdots\right]$. Therefore, any solution of

$$
\mathbf{A}_{\mathbf{r}} Y=-\lambda Y \quad(\lambda \in \mathbf{R})
$$

can exhibit the following two linearly independent expansions as $s \rightarrow 0: Y_{1} \sim 1$ and $Y_{2} \sim s^{1-\mu}$. We have that $Y_{1} \in L_{\rho}^{2}$ if $p<\bar{p}=(N+1) /(N-3)>p_{S}$, and $Y_{2} \notin L_{\rho}^{2}$ provided that $\mu \geq 3$, i.e., $p \leq p_{2}=(N+5) /(N+1)<p_{1}$. In this case the endpoint $\xi=1$ is in the limit-point case and we arrive at the following result.

Proposition 2.3. (i) For $p \in\left(1, p_{2}\right]$, the self-adjoint operator $\mathbf{A}_{\mathbf{r}}$ in $L_{\rho}^{2}\left(B_{1}\right)$ has a purely discrete countable spectrum of real simple eigenvalues

$$
\sigma\left(\mathbf{A}_{\mathbf{r}}\right)=\left\{\lambda_{k}=2 k(2 k+1+2 \alpha)+\alpha(1+\alpha), \quad k=0,1,2, \ldots\right\},
$$

the corresponding eigenfunctions $\psi_{k}$ are $2 k$ th order polynomials, and $\left\{\psi_{k}\right\}_{k \geq 0}$ form an orthonormal basis. 
(ii) If $p \in\left(p_{2}, p_{1}\right]$, then $\xi=1$ is in the limit-circle case, and the properties in (i) remain valid for symmetric functions satisfying the extra boundary condition as $\xi \rightarrow 1$ :

$$
(1-\xi)^{\nu} \theta(\xi) \rightarrow 0(\nu>0), \quad|\ln (1-\xi)|^{-1} \theta(\xi) \rightarrow 0(\nu=0) .
$$

(iii) If $p>p_{1}$, then the discrete spectrum (2.16) exists under the condition

$$
\psi, \psi^{\prime} \text { are bounded as } \xi \rightarrow 1 \text {. }
$$

Proof. For any $\lambda \in \mathbf{R}$, the symmetric solutions of Eq. (2.15) with analytic coefficients in $B_{1}$ are given by Kummer's series [3]

$$
\psi(\xi)=\sum_{k=0}^{\infty} C_{2 k} \xi^{2 k}
$$

Substituting into the equation, we obtain a recursion on the expansion coefficients

$$
C_{2 k+2}=A_{k} C_{2 k}, \quad k=1,2, \ldots,
$$

where

$$
A_{k}=\frac{2 k(2 k+1+2 \alpha)+\alpha(1+\alpha)-\lambda}{2(k+1)(2 k+N)}=1+\frac{a_{1}}{k}+O\left(\frac{1}{k^{2}}\right), k \rightarrow \infty,
$$

and $a_{1}=(2 \alpha-N-1) / 2$. It follows that this series converges uniformly on any interval $[0,1-\varepsilon]$ bounded away from 1 and is not uniformly converging on $[0,1]$. Moreover, we have that at the endpoint $\xi=1$, for $k \gg 1$, there holds $C_{2 k}=O\left(k^{-1}\right)$ with no sign changes if $p \neq p_{2}$ and $C_{2 k}=1+O\left(k^{-2}\right)$ if $p=p_{2}$. Hence, the series diverges at $\xi=1$ unless $\lambda=\lambda_{k}$ so that $A_{j}=0$ for all $j \geq k$. The corresponding eigenfunctions $\psi_{k}$ are $2 k$ th order polynomials

$$
\psi_{k}(\xi)=c_{k}\left(1+a_{1} \xi^{2}+\cdots+a_{k} \xi^{2 k}\right)
$$

where in view of (2.21) the coefficients change sign and each $\psi_{k}$ has $k$ zeros as in the Sturm-Liouville theory [29]. In both cases (ii) and (iii), extra boundary conditions at the degeneracy point $\xi=1$ follow from the above asymptotic analysis. Similar to the classical Hermite polynomials, the higher order term $\psi_{k}(\xi) \sim \xi^{2 k}$ gives the spectrum (2.16). The rest of the analysis is standard for self-adjoint second-order ordinary differential operators [29].

The first two $\left\{\lambda_{k}, \psi_{k}\right\}$ pairs are $\lambda_{0}=\alpha(1+\alpha), \psi_{0}(\xi)=c_{0}>0$ and

$$
\lambda_{1}=2(3+2 \alpha)+\alpha(1+\alpha), \quad \psi_{1}(\xi)=c_{1}\left[1-(2 \alpha+3) \xi^{2} / N\right] .
$$

Here $c_{0}, c_{1}, \ldots, c_{k}, \ldots$ denote normalization constants.

It is important that the polynomials $\left\{\psi_{k}(\xi)\right\}$ are defined globally in $\mathbf{R}_{+}$, i.e., inside the rescaled light cone $\{\xi<1\}$ and also outside it for $\xi>1$, so that $\left\{\psi_{k}\right\}$ are polynomial solutions of the problem posed in the complement $\{\xi>1\}$ with "eigenvalues" $\left\{\lambda_{k}\right\}$ prescribed by the inner eigenvalue problem. 
2.5. Internal variational problem: the first pattern. Let $f(\theta)=|\theta|^{p}$. Consider the following functional:

$$
F(v)=-\frac{1}{2} \int a(\xi)\left(v^{\prime}\right)^{2}-\frac{1}{2} \alpha(1+\alpha) \int v^{2}+\frac{1}{p+1} \int \rho|v|^{p} v .
$$

We will study its conditional critical points on the unit sphere in the Hilbert space of real-valued functions

$$
H_{a}^{1}((0,1))=\left\{v:\|v\|_{a}^{2}=\int a(\xi)\left(v^{\prime}\right)^{2}+\int v^{2}<\infty\right\},
$$

endowed with the corresponding inner product. This functional changes sign, is not uniform, and the corresponding operator of the Euler equation $\mathbf{A}_{\mathbf{r}}=F^{\prime}$ is non-coercive. In order to apply the fibering method [39], [40], we need embedding estimates.

Proposition 2.4. The embedding $H_{a}^{1}\left(B_{1}\right) \subset L_{\rho}^{p+1}\left(B_{1}\right)$ is compact if and only if

$$
\begin{gathered}
1<p<p_{S}=(N+2) /(N-2), \text { and } \\
1<p<1+2 / \nu, \nu>0(p \in(1, \infty) \text { if } \nu=0) .
\end{gathered}
$$

Proof. The first condition (2.23) is indeed coming from the standard analysis of the operator in a small neighbourhood of the origin $\xi=0$ and it is the same as for the semilinear elliptic operator $\Delta \theta+|\theta|^{p}$ (see [38]) so that $p_{S}$ is the Sobolev critical exponent. The second one $(2.24)$ is derived in a similar way by using the expansions of the coefficients $a$ and $\rho$ for $\xi \approx 1$.

Substituting $\nu \geq 0$ given in (2.12) into (2.24), one can see that it is always true provided $\nu \geq 0$, i.e., $p \leq p_{1}<p_{S}$. Thus, $1<p \leq p_{1}$ is necessary and sufficient condition for compact embedding of the functional spaces. We then apply the fibering method [39], [40] to prove the following result.

Theorem 2.1. Let $p \in\left(1, p_{1}\right]$. Then Eq. (2.7) with $f(\theta)=|\theta|^{p}$ admits a weak solution $\theta_{1} \in H_{a}^{1}((0,1))$, which is a bounded continuous function on $[0,1]$, positive on $[0,1)$.

As usual, the positivity property follows from the variational statement of the elliptic (ODE) problem. One can expect that Theorem 2.1 establishes existence of the constant weak solution $\theta_{1}$ given in (2.8).

2.6. On a countable spectrum of internal self-similar patterns. It is essential that Theorem 2.1 establishes existence of a single nonnegative self-similar profile $\left(\theta_{1}\right)$ for the hyperbolic equation (1.1). In general, the corresponding elliptic ODE (2.7) with nonmonotone nonlinearity $|\theta|^{p}$ admits no other nontrivial solutions; see examples in [40]. Consider the SWE (1.1). Looking for self-similar solutions (2.2), we arrive at the ODE

$$
\mathbf{A}_{\mathbf{r}} \theta \equiv\left(1-\xi^{2}\right) \theta^{\prime \prime}+\frac{N-1}{\xi} \theta^{\prime}-2(1+\alpha) \theta^{\prime} \xi-\alpha(1+\alpha) \theta=-|\theta|^{p-1} \theta
$$

for $\xi \in(0,1)$, admitting similar functional setting. Several results remain valid for this new equation. Nevertheless, there exists an important difference. Namely, in the corresponding functional

$$
F(v)=-\frac{1}{2} \int a(\xi)\left(v^{\prime}\right)^{2}-\frac{1}{2} \alpha(1+\alpha) \int v^{2}+\frac{1}{p+1} \int \rho|v|^{p+1}, \quad v \in H_{a}^{1},
$$


the last potential is homogeneous and nonnegative and both linear and nonlinear operators are monotone. Using the fibering method, we reduce the problem to the study of relative critical points of a homogeneous nonnegative functional on the unit sphere and the Lyusternik-Shnirel'man theory applies; see [39]. We then arrive at the following result.

TheOREM 2.2. For $p \in\left(1, p_{1}\right],(2.25)$ admits a countable spectrum of weak self-similar solutions $\left\{\theta_{k}(\xi), k=1,2, \ldots\right\}$, where $\theta=\theta_{k} \in H_{a}^{1}((0,1))$ satisfy $\left\|\theta_{k}\right\|_{a} \rightarrow \infty$ as $k \rightarrow \infty$.

As usual for such ODEs, the first solution $\theta_{1} \equiv c_{*}$ is positive in the non-degeneracy domain $(0,1)$ unlike the other profiles $\theta_{k}(\xi)$ which change sign exactly $k$ times in $(0,1)$.

Remark: nonexistence of a VSS for the wave equation with monotone operator. Similarity VSSs were first constructed for the semilinear parabolic equation with absorption

$$
u_{t}=\Delta u-|u|^{p-1} u, \quad 1<p<1+2 / N
$$

see [6], [12], and Chapter 2 in [43]. For a similar hyperbolic equation

$$
u_{t t}=\Delta u-|u|^{p-1} u
$$

with the same monotone operator on the right-hand side, such a construction of the symmetric VSS (2.2) leads to the stationary equation

$$
\mathbf{A}_{r} \theta-|\theta|^{p-1} \theta \equiv \frac{1}{\rho}\left[\left(a \theta^{\prime}\right)^{\prime}-\alpha(1+\alpha) \rho \theta\right]-|\theta|^{p-1} \theta=0 .
$$

One can see that the ODE operator is monotone, the equation admits a unique solution $\theta \equiv 0$ in $H_{a}^{1}$, and we arrive at the nonexistence result.

Proposition 2.5. The SWE (2.27) with $p \in\left(1, p_{1}\right]$ does not admit a nontrivial weak VSS.

3. Self-similar solutions for $p_{1}<p<p_{S}$. In the parameter range (1.3), the embedding is not compact and the variational method does not apply. In order to construct global weak continuous solutions $\theta(\xi)$, we will use a shooting argument.

3.1. Construction inside the light cone. We need some local properties of the solutions of the ODE (2.25) near the singularity point $\xi=1$. The asymptotic expansion essentially changes when $p$ passes through the critical exponent $p_{1}$. Such local solvability results and expansions are established by the Banach Contraction Principle applied to the equivalent integral equation. Proofs of a variety of similar asymptotic expansions as well as of global results can be found in [24].

Proposition 3.1. (i) If $1<p<p_{1}$ then (2.25); then, as $\xi \rightarrow 1-0$,

$$
\theta(\xi)=(C+o(1))(1-\xi)^{\omega}
$$

where, for $1<p<p_{3}=(N+1) /(N-1)$,

$$
\omega=-1 /(p-1), \quad C^{p-1}=[2 /(p-1)-N+1] /(p-1)>0,
$$

and, for $p_{3}<p<p_{1}, C \in \mathbf{R}$ is arbitrary and $\omega=-\nu=(N-1) / 2-\alpha<0$. For any $\theta_{0} \in \mathbf{R}$ there exists a unique solution with a regular expansion as $\xi \rightarrow 1-0$ corresponding 
to $C=0$ in $(3.1)$ :

$$
\begin{gathered}
\theta(\xi)=\theta_{R}(\xi) \equiv \theta_{0}+\gamma_{0}(1-\xi)+\delta_{0}(1-\xi)^{2}+\ldots \\
\gamma_{0}=G\left(\theta_{0}\right) / 2(1+\nu), \quad \delta_{0}=\nu^{-1}\left[\nu+N-G^{\prime}\left(\theta_{0}\right) G\left(\theta_{0}\right) / 4(1+\nu)\right],
\end{gathered}
$$

where $G(\theta)=f(\theta)-\alpha(1+\alpha) \theta$.

(ii) If $p_{1}<p<p_{4}=(N+1) /(N-3)>p_{S}$, then all orbits are bounded near $\xi=1$, and for any fixed $\theta_{0}=\theta(1) \in \mathbf{R}$, as $\xi \rightarrow 1-0$,

$$
\theta(\xi)=\theta_{R}(\xi)+\left[C(1-\xi)^{-\nu}+\cdots\right]
$$

where the regular expansion $\theta_{R}$ is as given in (3.2) and the term in square brackets describes a rational bundle with exponent $0<-\nu<1$ and arbitrary $C \in \mathbf{R}$.

It follows from (ii) that in this parameter range, the point $\xi=1$ is a removable singularity and the orbits pass through it, staying uniformly bounded and continuous. The result in (i) shows that it is not the case for $p<p_{1}$. We now prove the main result of this section.

TheOREM 3.1. Let $p_{1}<p<p_{S}$. Then there exists a smooth solution $\theta^{*}(\xi)>0$ of the $O D E(2.25)$ on $(0,1)$ satisfying $\left(\theta^{*}\right)^{\prime}(0)=0$ and $\theta^{*}(1)=0$. The extension $\theta^{*}(\xi) \equiv 0$ for $\xi>1$ determines a continuous weak solution of $(2.25)$ in $\mathbf{R}_{+}$.

Proof. Given arbitrary value $\mu \in \mathbf{R}$, denote by $\theta_{\mu}(\xi)$ the unique local in $\xi>0$ solution of (2.7) satisfying $\theta_{\mu}(0)=\mu$. Since the behaviour of $\theta_{\mu}$ for $\mu \gg 1$ near the origin is governed by the elliptic operator $\Delta \theta+\theta^{p}$ in the subcritical range $p<p_{S}$, by a scaling argument (see p. 188 in [43]) we conclude that

$$
\theta_{\mu}(\xi) \text { vanishes on }(0,1) \text { if } \mu \gg 1 \text {. }
$$

The function $\theta_{\mu}(\xi)$ depends continuously on $\mu$ on any interval $[0,1-\varepsilon], \varepsilon>0$, where Eq. (2.7) is not degenerate. On the other hand, since $\xi=1$ is a removable singularity and the orbits stay continuous at the singularity, by a standard argument we conclude that such a continuous dependence is available on the closed interval $[0,1]$.

Consider now $\theta_{\mu}$ for $\mu \approx c_{*}+0$, where such a behaviour is described by the linearized equation $\theta_{\mu}(\xi)=c_{*}+\left(\mu-c_{*}\right) Y(\xi)$. To the first approximation, $Y$ solves the linear equation

$$
\left(\mathbf{A}_{\mathbf{r}}+p \alpha(1+\alpha) I\right) Y=0, \quad \xi>0 ; \quad Y(0)=1, \quad Y^{\prime}(0)=0
$$

cf. [43], p. 191. By Proposition 2.3 we have that the first two eigenvalues of the self-adjoint operator $\mathbf{A}_{\mathbf{r}}+p \alpha(1+\alpha) I$ satisfy $\tilde{\lambda}_{0}=-(p-1) \alpha(1+\alpha)<0$ and $\tilde{\lambda}_{1}=4 p /(p-1)>0$. Therefore $Y$ has a unique zero on $(0,1)$. By the continuous dependence, this means that

$$
\theta_{\mu}(1) \rightarrow c_{*} \pm 0 \text { as } \mu \rightarrow c_{*} \mp 0 .
$$

It follows from (3.4) and (3.5) that there exists a $\mu^{*}>c_{*}$ such that $\theta_{\mu^{*}}(1)=0$. Then $\theta^{*} \equiv \theta_{\mu^{*}}$ has the behaviour (3.3) with $\theta_{0}=0$ near the singularity and the trivial extension for $\xi>1$ gives a weak solution of (2.7).

In order to complete the analysis of the orbits in $B_{1}$, we note that if $\mu \rightarrow 0$ then the linearized operator is $\mathbf{A}_{\mathbf{r}}$ with the positive spectrum, see Proposition 2.3. Therefore, for any sufficiently small $|\mu| \neq 0$ the function $\theta_{\mu}(\xi)$ does not change sign in $(0,1)$. Combining 
the above asymptotic properties and using the continuous dependence, we prove the following result which will be used later on for a suitable nontrivial extension of the solutions outside the light cone $\{\xi>1\}$.

Proposition 3.2. Let $p_{1}<p<p_{S}$. Given arbitrary $\theta_{0} \in \mathbf{R}$, there exists a solution $\theta(\xi)$ of $(2.25)$ such that $\theta(1)=\theta_{0}$.

Proof. We use a shooting argument with respect to parameter $C$ in (3.3). Due to well-known singularity properties of the radial elliptic operator $\Delta \theta+|\theta|^{p-1} \theta$ as $\xi \rightarrow 0$, we have that $\theta_{C}(\xi) \rightarrow+\infty$ as $\xi \rightarrow 0$ for $C \gg 1$ and $\theta_{C}(\xi) \rightarrow-\infty$ as $\xi \rightarrow 0$ for $C \ll-1$. By the continuous dependence on $C$, for some $C^{*}, \theta_{C^{*}}(\xi)$ is a bounded solution, hence satisfying the symmetry condition at the origin.

In particular, there exists a positive solution $\tilde{\theta}$ such that $\tilde{\theta}(1)=c_{*}$ and $\tilde{\theta}(0) \in\left(0, c_{*}\right)$. Extending it by $c_{*}$ in the supplement $\xi>1$, we obtain a nontrivial (not entirely flat) weak similarity solution $\tilde{u}(x, t)=t^{-\alpha} \tilde{\theta}(x / t)$ describing a weak singularity propagation concentrated on the light cone $\{|x|=t\}$.

3.2. Construction outside the light cone. Consider possible types of nontrivial extension of the solutions $\theta$ outside the light cone $\{\xi>1\}$. We consider the ODE (2.25) in $(1, \infty)$ in the parameter range $p_{1}<p<p_{S}$. Proposition 3.1 establishes that solutions are bounded at the singular endpoint $\xi=0$. Consider the right-hand singular endpoint $\xi=\infty$. The linearized operator is $\mathbf{A}_{\mathbf{r}}$. The homogeneous linearized equation $\mathbf{A}_{\mathbf{r}} Y=0$ admits two different types of solutions [24]:

$$
Y_{+}(\xi)=\xi^{-\alpha}+\ldots, \quad Y_{-}(\xi)=\xi^{-(p+1) /(p-1)}+\ldots, \quad \xi \rightarrow \infty .
$$

It is important that the initial traces of both self-similar solutions $u_{ \pm}(x, t)=t^{-\alpha} Y(\xi)=$ $t^{-\alpha} Y_{ \pm}(\xi)+\ldots$ as $t \rightarrow+0$ are quite different, $u_{+}(x,+0)=|x|^{-2 /(p-1)}$ and $u_{-}(x,+0)=0$ for $x \neq 0$. We now use a similar shooting technique and begin with the study of oscillatory properties of solutions of (2.25). Fix an arbitrary constant $\gamma$, and consider a family of solutions $\theta_{C}(\xi)$ of $(2.25)$ with the asymptotic behaviour

$$
\theta_{C}(\xi)=\gamma Y_{-}(\xi)+C Y_{+}(\xi)+\ldots, \quad \xi \rightarrow \infty ; \quad C \in \mathbf{R} .
$$

Let us study the limits $C \rightarrow \pm \infty$. We perform the scaling $\theta_{C}(\xi)=C Z(\xi)$ and hence for large $\xi, Z(\xi)=\xi^{-\alpha}+\cdots$ for all $|C| \gg 1$. Consider the corresponding perturbed equation for $Z$ :

$$
\xi^{2} Z^{\prime \prime}+2(1+\alpha) Z^{\prime} \xi+\alpha(1+\alpha) Z=|C|^{p-1}|Z|^{p-1} Z+\ldots, \quad \xi \gg 1 .
$$

It follows that for $C \gg 1$, there exists blowing up of the solution, $\theta_{C}(\xi) \rightarrow+\infty$ at a finite $\xi \rightarrow \xi_{C}+0$, where $\xi_{C} \rightarrow \infty$ as $C \rightarrow \infty$, with the singular behaviour given by the monotone operator $-\xi_{C}^{2} \theta^{\prime \prime}+|\theta|^{p-1} \theta$. Similarly, the solution blows up for any $C \ll-1, \theta_{C}(\xi) \rightarrow-\infty$ as $\xi \rightarrow \xi_{C}+0$, where $\xi_{C} \rightarrow \infty$ as $C \rightarrow-\infty$. By the continuity argument for second-order ODEs, it follows that there exists a $C_{*}=C_{*}(\gamma)$ such that the solution $\theta^{*}(\xi) \equiv \theta_{C_{*}}(\xi)$ does not blow-up on $(1, \infty)$ and is bounded at $\xi=1$ by Proposition 3.1, (ii). Hence, it is a bounded continuous solution defined for any $\xi \geq 1$ (such a construction is similar to that for quasilinear ODEs on p. 193 in [43]). Fixing $\theta_{0}=\theta^{*}(1)$, by Proposition 3.2 we can extend it inside the light cone $B_{1}$ and arrive at a global weak solution $\theta^{*}(\xi)$ in $\mathbf{R}_{+}$. Summing up, we obtain the following result. 
TheOrem 3.2. Let $p_{1}<p<p_{S}$. Then there exists a one-parametric family $\left\{\theta_{\gamma}^{*}\right\}$ of continuous weak solutions of the ODE (2.25) in $\mathbf{R}_{+}$.

We note that typical evolution approaches [35]-[37], [42] to construction of similarity solutions based on solvability of scaling-invariant Cauchy problems for the SWE deal with sufficiently small similarity profiles $\theta^{*}$ (indeed, large solutions, in general, blow-up in finite time).

4. Linearized countable spectra of blow-up patterns. In the hyperbolic equations, blow-up singularities propagate with finite speed and form a smooth blow-up surface in $\mathbf{R}^{N+1}$. Blow-up solutions admit a natural extension beyond blow-up singularity, and blow-up is always incomplete, unlike the scmilinear and quasilinear parabolic equations, where the problem of continuation beyond blow-up is not straightforward and essentially depends on nonlinearities involved; see [14] and references therein.

4.1. Self-similar occurrence of blow-up as first approximation. The phenomena of the propagation of blow-up singularities and properties of blow-up surfaces for equations like (1.1) are well-known; see [7], [8], [26], and Chapter 3 in [1]. Locally, the blow-up propagation is self-similar; see a comment below. The phenomenon of occurrence of blowup singularities from uniformly bounded solutions for SWEs is an important question of the general theory of nonlinear evolution equations of hyperbolic type; see results and references in [26] and [1].

We assume that the solution blows up first time at $t=-0$ at the origin $x=0$, so that it is uniformly bounded on any subset $\mathbf{R}^{N} \times[-1,-\varepsilon], \varepsilon>0$. Our main goal is to describe a spectrum of blow-up patterns near the point $x=0, t=-0$. For simplicity, we assume that the blow-up behaviour is radially symmetric, a typical blow-up assumption which makes it possible to study the blow-up singularity by using eigenfunctions and matching procedures based on an ODE analysis instead of the more general and technically more involved elliptic one for non-symmetric blow-up.

Using the invariant reflection transformation $t \mapsto-t$, we have that the blow-up selfsimilar solutions

$$
u_{*}(x, t)=(-t)^{-\alpha} \theta(\eta), \quad \eta=x /(-t),
$$

are governed by the same continuous profiles $\theta$ satisfying the elliptic equation (2.3) or its radial restriction (2.7). Existence results inside the (artificial) light cone $\{|\eta|<1\}$ on the global self-similar solutions in Theorems 2.1, 2.2, and in Sec. 2 coincide with those for the blow-up solutions.

We will show that the first blow-up pattern (obtained from $(2.9)$ by $t \mapsto-t$ )

$$
u_{1}(x, t)=(-t)^{-\alpha} c_{*},
$$

describes a generic occurrence of blow-up singularity from bounded symmetric initial data on compact subsets inside $|\eta|<1$ and outside $|\eta|>1$ of the light cone. Below, we show how to derive a refined countable spectrum of different positive patterns for this SWE by using a linearization and a nonlinear matching procedure. 
Remark: on self-similar blow-up propagation. The symmetric ODE (2.5) admits an exact singular solution in the open supplement $\{|\eta|>1\}$ of the unit ball:

$$
\theta_{S}(\eta)=C_{S}\left(|\eta|^{2}-1\right)_{+}^{-1 /(p-1)}>0, \quad C_{S}^{p-1}=2[N-1-2 /(p-1)] /(p-1),
$$

where $\theta_{S} \rightarrow \infty$ as $|\eta| \rightarrow+1$. It follows that such a singular solution exists in the supercritical range $p>p_{1}=(N+3) /(N-1)$. The corresponding self-similar solution $u_{S}(x, t)=t^{-2 /(p-1)} C_{S}\left[(|x| / t)^{2}-1\right]_{+}^{-1 /(p-1)}$ is known to describe a (generic) propagation of blow-up singularities on the moving sphere $\{|x|=t\}$; see general results in [7] and [8].

4.2. Countable spectrum of linearized patterns in the inner region. We recall that a countable subset of similarity blow-up patterns (4.1), constructed in Theorem 2.2 for the monotone nonlinear term, is composed from the profiles $\theta_{k}(\xi)$ changing sign for any $k \geq 2$. Hence these similarity solutions tend to $\pm \infty$ as $t \rightarrow-0$. Such single point nonuniform blow-up is expected to be unstable. In order to construct a spectrum of new positive patterns, we first consider the linearized problem describing possible types of approaching as $t \rightarrow-0$ of the evolution orbit $u(x, t)$ to the first pattern (4.2). We then introduce the corresponding rescaled variables by setting

$$
u(x, t)=(-t)^{-\alpha} g(\xi, \tau), \quad \tau=-\ln (-t) \rightarrow \infty \text { as } t \rightarrow-0,
$$

where the rescaled solution $g$ solves the semilinear hyperbolic equation

$$
\mathbf{P} g \equiv g_{\tau \tau}+(1+2 \alpha) g_{\tau}+2 g_{\tau \xi} \xi=\mathbf{A}_{\mathbf{r}} g+f(g),
$$

where, as usual, $f(g)=|g|^{p}$ or $f(g)=|g|^{p-1} g$. We assume that $g(\cdot, \tau) \rightarrow \theta_{1}(\cdot)=c_{*}$ as $\tau \rightarrow \infty$ uniformly on compact subsets. We next perform a standard linearization in Eq. (4.5) by setting

$$
g(\xi, \tau)=c_{*}+Y(\xi, \tau)
$$

and $Y$ solves the perturbed equation

$$
\mathbf{P} Y=\mathbf{C}_{\mathbf{r}} Y+\mathbf{D}(Y), \quad \mathbf{C}_{\mathbf{r}}=\mathbf{A}_{\mathbf{r}}+p \alpha(1+\alpha) I,
$$

where $\mathbf{A}_{\mathbf{r}}$ is given in (2.7) so that

$$
\mathbf{C}_{\mathbf{r}}=\left(1-\xi^{2}\right) \frac{d^{2}}{d \xi^{2}}+\frac{N-1}{\xi} \frac{d}{d \xi}-2(1+\alpha) \xi \frac{d}{d \xi}+\alpha(1+\alpha)(p-1) I .
$$

The perturbation term $\mathbf{D}$ is quadratic, $\mathbf{D}(Y)=O\left(Y^{2}\right)$ as $Y \rightarrow 0$. Spectral properties of self-adjoint extensions of operator $\mathbf{C}_{\mathbf{r}}$ inside the light cone are given in Propositions 2.3, where necessary functional setting is presented.

We apply a standard linear perturbation approach; see classical books [9] and [28]. Namely, we are looking for the asymptotic expansion of solutions of the perturbed equation

$$
Y(\xi, \tau)=C e^{-\mu \tau} \phi(\xi)+o\left(e^{-\mu \tau}\right)(C=\text { const } \neq 0),
$$

uniformly on any compact subset in $\xi$. Substituting (4.8) into Eq. (4.7) and keeping the main exponential terms, we arrive at the following eigenvalue problem:

$$
\mathbf{C}_{\mathbf{r}} \phi=\mu^{2} \phi-\mu(1+2 \alpha) \phi-2 \mu \phi^{\prime} \xi \text { in } \mathbf{R}_{+},
$$

for a quadratic pencil of linear operators; see a general theory in [31]. At the stage of formal asymptotic expansion theory, we avoid a detailed functional setting for such a 
quadratic pencil, and note that for the ordinary differential operators such eigenvalue problems are well-known; see, e.g., Sec. 2 in [34]. A proper functional setting follows from the above analysis of self-adjoint operators $\mathbf{A}_{\mathbf{r}}$ and $\mathbf{C}_{\mathbf{r}}$. At the crucial singular point $\xi=1 \pm 0$, we consider the operators in $L_{\rho}^{2}$ with the domain $H_{a}^{2}$ of symmetric functions satisfying necessary conditions at singular endpoints. As a natural requirement of the extension problem outside the light cone $\{\xi>1\}$, we cannot impose conditions at the singularity point $\xi=\infty$ and moreover consider operators in a weighted space $L_{\bar{\rho}}^{2}$ with exponentially fast decaying weight $\bar{\rho}(\xi) \sim e^{-b|\xi|^{2}}$ as $\xi \rightarrow \infty, b>0$ is a constant. Let us state the result.

Proposition 4.1. For $p>1$, the eigenvalue problem (4.9) admits two spectra $\left\{\phi_{k}^{ \pm}\right\}_{k \geq 0}$ of analytic eigenfunctions, which are $2 k$ th order polynomials, corresponding to the eigenvalues

$$
\mu_{k}^{-}=2 k-1, \quad \mu_{k}^{+}=2 k+2(p+1) /(p-1), \quad k=0,1,2, \ldots
$$

Proof. As in the proof of Proposition 2.3, using Kummer's series in the construction of analytic solutions, we arrive at the recursion (2.20) with the coefficient

$$
A_{k}=\frac{2 k[2 k+1+2 \alpha-2 \mu]+(p-1) \alpha(1+\alpha)-\mu^{2}+\mu(1+2 \alpha)}{2(k+1)(2 k+N)} .
$$

The truncation condition of the series implies the following quadratic equation on the eigenvalues $\mu=\mu_{k}$ :

$$
\mu^{2}-\mu(1+2 \alpha+4 k)-(p-1) \alpha(1+\alpha)+2 k(2 k+1+2 \alpha)=0,
$$

whence the result. The $2 k$ th order polynomials $\phi_{k}^{ \pm}(\xi)$ are well-defined for all $\xi \geq 0$.

The positive eigenvalues

$$
\mu_{k}^{+}>0, \quad k=0,1,2, \ldots ; \quad \mu_{k}^{-}>0, \quad k=1,2, \ldots,
$$

correspond to the stable subspace of the origin for the linear operator on the righthand side of (4.7). There exists a single negative eigenvalue $\mu_{0}^{-}=-1$ with $\phi_{0}^{-}=$const describing a linear instability. Obviously, this one-dimensional unstable subspace is due to the time translational invariance of the semilinear equation (corresponds to shifting of the blow-up time). Therefore, the first blow-up pattern $\theta_{1}=c_{*}$ is stable in the linear approximation.

Each subset of $2 k$ th order polynomials is complete in weighted spaces like $L_{\rho}^{2}\left(\mathbf{R}_{+}\right)$of symmetric functions; see, e.g., [27].

4.3. Unstability of the uniform global pattern. It follows from the above linearized analysis that the uniform profile $\theta_{1} \equiv c_{*}$ studied in the previous section is unstable in the class of global solutions defined for all $t>0$. Indeed, introducing the rescaled variables (cf. (4.4))

$$
u(x, t)=t^{-\alpha} g(\xi, \tau), \quad \xi=x / t, \quad \tau=\ln t,
$$

we arrive at the equation (cf. (4.5))

$$
\mathbf{P} g \equiv g_{\tau \tau}-(1+2 \alpha) g_{\tau}-2 g_{\tau \xi} \xi=\mathbf{A}_{r} g+f(g),
$$


where linear operators containing the first-order derivative $d / d \tau$ change sign. Via linearization (4.6), this leads to the eigenvalue problem (cf. (4.9))

$$
\mathbf{C}_{\mathbf{r}} \phi=\mu^{2} \phi+\mu(1+2 \alpha) \phi+2 \mu \phi^{\prime} \xi
$$

and to the following quadratic equation for the eigenvalues (cf. (4.12)):

$$
\mu^{2}+\mu(1+2 \alpha+4 k)-(p-1) \alpha(1+\alpha)+2 k(2 k+1+2 \alpha)=0,
$$

with the opposite sign in the second term. This implies that all $\mu_{k}^{ \pm}<0$ (unstability) except the single one, $\mu_{0}^{-}>0$, describing the trivial stability of the self-similar behaviour as $t \rightarrow \infty$ under the time-translation $t \mapsto t+T$.

4.4. Transition to the intermediate $O D E$ region: a perturbed dynamical system. In order to extend expansion (4.8) with any $\mu=\mu_{k}^{-}>0$ and $\phi=\phi_{k}^{-}$into the nonlinear intermediate region to be specified later on, we use the fact that each eigenfunction $\phi_{k}(\xi)$ is a polynomial of the order $2 k$. Hence, for $\xi \gg 1$, the first term in (4.8) written in the form

$Y_{k}(\xi, \tau)=C e^{-\mu_{k} \tau} \phi_{k}(\xi)+\cdots=C c_{k} e^{-\mu_{k} \tau} \xi^{2 k}+\cdots=C c_{k}\left(e^{-\beta_{k} \tau} \xi\right)^{2 k}+\cdots \equiv C c_{k} \zeta^{2 k}+\ldots$,

where $\beta_{k}=\mu_{k} / 2 k$ and $c_{k}$ denote the normalization constants, suggests an extra scaling $\zeta=e^{-\beta_{k} \tau} \xi$. Then Eq. (4.5) for the rescaled function $h(\zeta, \tau)=g\left(\zeta e^{\beta_{k} \tau}, \tau\right)$ reduces to the following perturbed one:

$$
\mathbf{P} h-2 \beta_{k} h_{\tau \zeta} \zeta=\mathbf{D}_{\mathbf{k}} h+|h|^{p-1} h+e^{-2 \beta_{k} \tau} \Delta_{\zeta} h,
$$

with the linear ordinary differential operator

$$
\mathbf{D}_{\mathbf{k}} h=-\left(\beta_{k}-1\right)^{2} h_{\zeta \zeta} \zeta^{2}+\left(\beta_{k}-1\right)\left[2(1+\alpha)-\beta_{k}\right] h_{\zeta} \zeta-\alpha(1+\alpha) h .
$$

It is important that in these rescaled variables, on sufficiently smooth evolution orbits, the Laplace operator $\Delta u$ in the hyperbolic equation reduces to an exponentially small perturbation that is negligible as $\tau \rightarrow \infty$, i.e., $t \rightarrow-0$. This means that in the intermediate region for $t \approx-0$, the smooth global flow is governed by the second-order ODE

$$
u_{t t}=|u|^{p-1} u \text {. }
$$

As above, we first study the corresponding elliptic (stationary) equation obtained by the passage to the limit $\tau \rightarrow \infty$ in (4.19) under the natural regularity assumptions on smooth compact orbits:

$$
\mathbf{D}_{\mathbf{k}} H+|H|^{p-1} H=0 \text {. }
$$

The function $H$ is the rescaled self-similar solution of the ODE (4.21). In the radial case, it is Euler's equation with a nonlinear term. Setting $y=\ln \zeta$, we arrive at the autonomous second-order ODE

$$
\left(\beta_{k}-1\right)^{2} H^{\prime \prime}-\left(\beta_{k}-1\right)(2 \alpha+1) H^{\prime}+\alpha(1+\alpha) H-|H|^{p-1} H=0, \quad y \in \mathbf{R} .
$$

It reduces to a first-order ODE and a standard phase-plane analysis applies. It admits a one-parameter family of profiles

$$
H_{D}(\zeta)=c_{*}\left(1+D \zeta^{\nu_{k}}\right)^{-2 /(p-1)}, \quad \nu_{k}=1 /\left(1-\beta_{k}\right),
$$


where $D>0$ is a parameter. We choose all appropriate eigenvalue-eigenfunction pairs $\left\{\mu_{k}^{-}, \phi_{k}^{-}\right\}$corresponding to the occurrence of blow-up from bounded classical solutions. Then $\beta_{k}=\mu_{k}^{-} / 2 k=1-1 / 2 k<1$, and (4.24) with exponent $\nu_{k}>0$ gives a monotone decreasing function $H_{D}(\zeta) \rightarrow 0$ as $\zeta \rightarrow \infty$. This makes it possible to match such a localized blow-up behaviour with smooth bounded flow beyond the blow-up set. Substituting $\beta_{k}$ into (4.24), we get the profiles

$$
H_{D}(\zeta)=c_{*}\left(1+D \zeta^{2 k}\right)^{-2 /(p-1)}
$$

The matching condition of (4.25) as $\zeta \rightarrow 0$ and (4.18) is $D=-(p-1) c_{k} C / 2 c_{*}$. This uniquely chosen profile $H_{D}(\zeta)$ gives the desired behaviour of the solution in the intermediate region.

4.5. Transition to the outer region, a spectrum of final-time profiles. Let us extend the behaviour in the intermediate ODE region into the outer region where $0<|x| \ll 1$. We use the asymptotic behaviour of $H_{D}(\zeta)$ as $\zeta \rightarrow \infty$. Such type of extension was established for a wide class of quasilinear second-order parabolic equations; see [5], [18] and Sec. 8 in [13]. It follows from (4.25) that

$$
H_{D}(\zeta)=c_{*} D^{-2 /(p-1)} \zeta^{-4 k /(p-1)}+\ldots, \quad \zeta \rightarrow \infty, \quad k=1,2, \ldots .
$$

Since $\zeta=(-t)^{\beta_{k}} \xi=(-t)^{\beta_{k}-1}|x|$, we then obtain, passing to the limit $t \rightarrow-0$, that the asymptotic behaviour in the outer region inherits (by extension) the behaviour in the neighbour intermediate one,

$$
u(x, t)=(-t)^{-\alpha} H_{D}(\zeta)(1+o(1))=(-t)^{-\alpha} c_{*} D^{-2 /(p-1)}\left((-t)^{\beta_{k}-1}|x|\right)^{-4 k /(p-1)}+\ldots,
$$

where the exponent of $(-t)$ vanishes, $-\alpha-4 k\left(\beta_{k}-1\right) /(p-1)=0$, so that $u(x,-0)=$ $c_{*} D^{-2 /(p-1)}|x|^{-4 k /(p-1)}+\ldots$ The countable set

$$
\left\{|x|^{-4 k /(p-1)}, \quad k=1,2, \ldots\right\}
$$

determines possible types of asymptotics of final-time profiles near the singular point $x=0$. Then $k=1$ is expected to correspond to the stable (generic) blow-up pattern, while for $k=2,3, \ldots$ such final-time profiles correspond to more flat unstable blow-up, when the orbit blowing-up at $x=0, t=-0$ originates an analytic blow-up surface on the $\{x, t\}$-plane with the local behaviour of the type $t=\psi_{k}(x)=a_{0}+b_{0} x^{2 k}+\ldots, a_{0}, b_{0}>0$, with $x=0$ being a local minimum.

Acknowledgements. The second author thanks the Department of Mathematical Sciences of the University of Bath for its hospitality during his visit sponsored by a grant from the EPSRC.

\section{REFERENCES}

[1] S. Alinhac, Blow-up for Nonlinear Hyperbolic Equations, Birkhäuser, Boston/Berlin, 1995

[2] D. Amadori, Unstable blow-up patterns, Differ. Integr. Equat., 8, 1977-1996 (1995)

[3] H. Bateman and E. Erdélyi, Higher Transcendental Functions, Vol. I, McGraw-Hill, New York, 1953

[4] M. S. Birman and M. Z. Solomjak, Spectral Theory of Self-Adjoint Operators in Hilbert Space, D. Reidel, Dordrecht/Tokyo, 1987

[5] A. Bressan, Stable blow-up patterns, J. Differ. Equat., 98, 57-75 (1992)

[6] H. Brezis, L. A. Peletier, and D. Terman, A very singular solution of the heat equation with absorption, Arch. Rat. Mech. Anal., 95, 185-209 (1986) 
[7] L. A. Caffarelli and A. Friedman, Differentiability of the blow-up curve for one dimensional nonlinear wave equations, Arch. Rational Mech. Anal., 91, 83-98 (1985)

[8] L. A. Caffarelli and A. Friedman, The blow-up boundary for nonlinear wave equations, Trans. Amer. Math. Soc., 297 223-241 (1986)

[9] Ju. L. Daleckii and M. G. Krein, Stability of Solutions of Differential Equations in Banach Space, Transl. Math. Monographs, Vol. 43, Amer. Math. Soc., Providence, RI, 1974

[10] S. Filippas and R. V. Kohn, Refined asymptotics for the blow-up of $u_{t}-\Delta u=u^{p}$, Comm. Pure Appl. Math., 45, 821-869 (1992)

[11] H. Fujita, On the blowing up of solutions to the Cauchy problem for $u_{t}=\Delta u+u^{1+\alpha}$, J. Fac. Sci. Univ. Tokyo, Sect. IA, Math., 13, 109-124 (1966)

[12] V. A. Galaktionov, S. P. Kurdyumov, and A. A. Samarskii, On asymptotic "eigenfunctions" of the Cauchy problem for a nonlinear parabolic equation, Math. USSR Sbornik, 54, 421--455 (1986)

[13] V. A. Galaktionov and J. L. Vazquez, Blow-up for quasilinear heat equations described by means of nonlinear Hamilton-Jacobi equations, J. Differ. Equat., 127, 1-40 (1996)

[14] V. A. Galaktionov and J. L. Vazquez, Continuation of blow-up solutions of nonlinear heat equations in several space dimensions, Comm. Pure Appl. Math., 50, 1-68 (1997)

[15] V. Georgiev, H. Lindblad, and C. Sogge, Weighted Strichartz estimates and global existence for semilinear wave equations, Amer. J. Math., 119, 1291-1319 (1997)

[16] H. P. Heinig, Weighted norm inequalities for classes of operators, Indiana Univ. Math. J., 33, 573-582 (1984)

[17] D. B. Henry, Some infinite-dimensional Morse-Smale systems defined by parabolic partial differential equations, J. Differ. Equat., 59, 165-205 (1985)

[18] M. A. Herrero and J. J. L. Velázquez, Blow-up behaviour of one-dimensional semilinear parabolic equations, Ann. Inst. Henri Poincaré, Analyse non linéaire, 10, 131-189 (1993)

[19] L. Hörmander, Lectures on Nonlinear Hyperbolic Differential Equations, Math. and Appl., Vol. 26, Springer-Verlag, Berlin/New York, 1997

[20] F. John, Blow-up of solutions of nonlinear wave equation in three space dimensions, Manuscripta Math., 28, 235-268 (1979)

[21] S. Kamin and L. A. Peletier, Large time behaviour of solutions of the heat equation with absorption, Ann. Sc. Norm. Pisa Cl. Sci. (4), 12, 393-408 (1984)

[22] S. Kamin and L. A. Peletier, Large time behaviour of solutions of the porous media equation with absorption, Israel J. of Math., 55, 129-146 (1986)

[23] T. Kato, Blow-up of solutions of some nonlinear hyperbolic equations, Comm. Pure Appl. Math., 32, 501-505 (1980)

[24] O. Kavian and F. B. Weissler, Finite energy self-similar solutions of a nonlinear wave equation, Comm. Partial Differ. Equat., 15, 1381-1420 (1990)

[25] J. Keller, On solutions of nonlinear wave equations, Comm. Pure Appl. Math., 10, 523-530 (1957)

[26] S. Kichenassamy and W. Littman, Blow-up surfaces for nonlinear wave equations, I and II, Comm. Partial Differ. Equat., 18, 431-452, and 1869-1899 (1993)

[27] A. N. Kolmogorov and S. V. Fomin, Elements of the Theory of Functions and Functional Analysis, Nauka, Moscow, 1976

[28] S. G. Krein, Linear Differential Equations in Banach Space, Transl. Math. Monographs, Vol. 29, Amer. Math. Soc., Providence, RI, 1971

[29] B. M. Levitan and I. S. Sargsjan, Introduction to Spectral Theory: Self-Adjoint Ordinary Differential Operators, Transl. Math. Mon., Vol. 39, Amer. Math. Soc., Providence, RI, 1975

[30] H. Lingblad and C. Sogge, On existence and scattering with minimal regularity for semilinear wave equations, J. Differ. Equat., 130, 357-426 (1995)

[31] A. S. Markus, Introduction to the Spectral Theory of Polynomial Operator Pencils, Transl. Math. Mon., Vol. 71, Amer. Math. Soc., Providence, RI, 1988

[32] F. Merle and H. Zaag, Stability of the blow-up profile for equations of the type $u_{t}=\Delta u+|u|^{p-1} u$, Duke Math. J., 86, 143-195 (1997)

[33] E. Mitidieri and S. I. Pohozaev, A Priori Estimates and Blow-up of Solutions to Nonlinear Partial Differential Equations and Inequalities, Proc. Steklov Math. Inst., 3, Vol. 234, Moscow, 2001 (ISSN:0081-5438)

[34] M. A. Naimark, Linear Differential Operators, Part 1, Frederick Ungar Publ. Co., New York, 1967

[35] H. Pecher, Sharp existence results for self-similar solutions of semilinear wave equations, Nonl. Differ. Equat. Appl., 7, 323-341 (2000) 
[36] H. Pecher, Self-similar and asymptotically self-similar solutions of nonlinear wave equations, Math. Ann., 316, 259-281 (2000)

[37] F. Planchon, Self-similar solutions and semi-linear wave equations in Besov spaces, J. Math. Anal. Appl., 78, 809-820 (2000)

[38] S. I. Pohozaev, Eigenfunctions of the equation $\Delta u+\lambda f(u)=0$, Soviet Math. Dokl., 6, 1408-1411 (1965)

[39] S. I. Pohozaev, On an approach to nonlinear equations, Soviet Math. Dokl., 20, 912-916 (1979)

[40] S. I. Pohozaev, The fibering method in nonlinear variational problems, Pitman Research Notes in Math., Vol. 365, Pitman, pp. 35-88 (1997)

[41] S. I. Pohozaev and L. Véron, Blow-up results for nonlinear hyperbolic inequalities, Annali Scuola Norm. Sup. Pisa, Ser. IV, 29, 393-420 (2000)

[42] F. Ribaud and A. Youssfi, Solutions globales et solutions auto-similaires de l'équation des ondes non linéaire, C. R. Acad. Sci. Paris Sér. I Math. 329, 33-36 (1999)

[43] A. A. Samarskii, V. A. Galaktionov, S. P. Kurdyumov, and A. P. Mikhailov, Blow-up in Quasilinear Parabolic Equations, Walter de Gruyter, Berlin/New York, 1995

[44] T. C. Sideris, Nonexistence of global solutions to semilinear wave equations in high dimensions, J. Differ. Equat., 32, 378-406 (1984)

[45] C. Sturm, Mémoire sur une classe d'équations à différences partielles, J. Math. Pures Appl., 1, 373-444 (1836)

[46] J. J. L. Velázquez, Estimates on $(N-1)$-dimensional Hausdorff measure of the blow-up set for a semilinear heat equation, Indiana Univ. Math. J., 42, 445-476 (1993)

[47] J. J. L. Velázquez, V. A. Galaktionov, and M. A. Herrero, The space structure near a blow-up point for semilinear heat equations: a formal approach, USSR Comput. Math. Phys., 31, 46-55 (1991) 